\title{
EXISTENTIALLY CLOSED STRUCTURES AND SOME EMBEDDING THEOREMS
}

\author{
M. SHAHRYARI
}

\begin{abstract}
Using the notion of existentially closed structures, we obtain embedding theorems for groups and Lie algebras. We also prove the existence of some groups and Lie algebras with prescribed properties.
\end{abstract}

\begin{abstract}
AMS Subject Classification Primary 20E45, Secondary 20 E06.
Key Words Algebraically closedness; Existentially closedness; Existentially completeness; Inductive classes; Axiom of choice; Embedding of groups and Lie algebras; HNN-extension.
\end{abstract}

An algebraic system $A$ is called existentially closed, if every consistent finite set of existential sentences with parameters from $A$, is satisfiable in $A$. Using the disjunctive normal form of existential sentences, one can easily see that $A$ is existentially closed, if and only if any consistent finite set of equations and in-equations with parameters from $A$, is satisfiable in $A$. As a special case, we can define the notion of existentially closed groups. It can be shown that (see [1]), a group $G \neq 1$ is existentially closed group, if and only if every consistent finite system of equations with parameters from $G$, has a solution in $G$. In the general case, an algebraic system with the property that every consistent finite set of equations has a solution in that algebraic system is called algebraically closed. So, in the case of groups (and Lie algebras), two properties of being existentially closed and being algebraically closed are equivalent.

The notion of existentially closed groups is introduced by a short paper of W. R. Scott in 1951, and he used the axiom of choice to prove the existence of such groups, [5]. Since then, the class of existentially closed groups is studied extensively and many interesting properties of such groups are discovered during the past decades. For a review of the history of existentially closed groups, the reader can see [1] or [3].

In this article, using the concept of existentially closed groups and Lie algebras, we prove some embedding theorems. For example, we will show that for any set of primes $\pi$, any infinite $\mathrm{T}_{\pi}$-group can be embedded in a

Date: June 3, 2021. 
simple $\pi^{\prime}$-divisible $\mathrm{T}_{\pi}$-group with the same cardinality. Further in the larger group, elements of the same orders are conjugate. By a $\mathrm{T}_{\pi}$-group, we mean a group $G$ such that the equality $x^{m}=1$ implies $x=1$, whenever $m$ is a $\pi^{\prime}$-number. By the notion of existentially closed Lie algebras, we show that any Lie algebra $L$ can be embedded in a simple Lie algebra $L^{*}$ which has many interesting properties; for example, for any non-zero elements $a$ and $b$, there is $x$ such that $[x, a]=b$. In the case of finite fields, every finite dimensional Lie algebra can be embedded in $L^{*}$ and it is possible to describe the derivation algebra of any finite dimensional algebra $A$ as the quotient algebra $N_{G^{*}}(A) / C_{G^{*}}(A)$. We also prove the existence of some groups and Lie algebras with prescribed properties. Our main tool in this work is HNNextensions of groups and Lie algebras. The first one is well-known and the reader can consult any book on combinatorial group theory (for example [3]) to see definition and properties of HNN-extensions of groups. The HNNextensions of Lie algebras are not so popular and it seems that there are only two articles ever published in the subject, [2] and [7].

Although, we are dealing just with groups and Lie algebras in this article, it is useful to give a general definition of existentially closed structures in the frame of the universal algebra. Let $\mathcal{L}$ be an algebraic language and $A$ be an algebra of type $\mathcal{L}$. We extend $\mathcal{L}$ to a new language $\mathcal{L}_{A}$ by adding new constant symbols $c_{a}$ for any $a \in A$. Let $T_{A}(X)$ be the term algebra of $\mathcal{L}_{A}$ with variables from a countable set $X$. If $p\left(x_{1}, \ldots, x_{n}\right)$ and $q\left(x_{1}, \ldots, x_{n}\right)$ are elements of this term algebra, then we call the expression $p\left(x_{1}, \ldots, x_{n}\right)=$ $q\left(x_{1}, \ldots, x_{n}\right)$ an equation with coefficients (or parameters) from $A$. An inequation is the negation of an equation. A system of equations and inequations over $A$ (or a system over $A$ ) is a finite set consisting equations and in-equations. We say that $A$ is existentially closed, if and only if any system over $A$ having a solution in an extension $B$ of $A$, has already a solution in $A$. In this article, we consider the case of groups and Lie algebras, but some of the theory here, can be generalized to arbitrary algebraic structures. Note that in the case of a group $G$, we may assume that an equation has the form $w\left(x_{1}, \ldots, x_{n}\right)=1$, where $w$ is an element of the free product $G * F(X)$. Here $F(X)$ is the free group on the set $X$. Similarly, if $L$ is a Lie algebra (or any non-associative algebra), then an equation over $L$ has the form $w\left(x_{1}, \ldots, x_{n}\right)=0$, with $w$ and element of the free product $L * F(X)$, where $F(X)$ is the free Lie algebra over $X$.

\section{Existentially ClOSED GROUPS}

A group $G$ is called existentially closed, if any finite consistent system of equations and in-equations with coefficients from $G$ has a solution in $G$. A system

$$
S=\left\{w_{i}\left(x_{1}, \ldots, x_{n}\right)=1 ;(1 \leq i \leq r), w_{j}\left(x_{1}, \ldots, x_{n}\right) \neq 1 ;(r+1 \leq j \leq s)\right\}
$$

with coefficients in $G$ is called consistent, if there is a group $K$ containing $G$, such that $S$ has a solution in $K$. One can generalize this definition to 
an arbitrary class of groups: Let $\mathfrak{X}$ be a class of groups. A group $G \in \mathfrak{X}$ is called existentially closed in the class $\mathfrak{X}$, if every $\mathfrak{X}$-consistent system $S$ has a solution in $G$. Here, $\mathfrak{X}$-consistency means that there exists a group $K \in \mathfrak{X}$ which contains $G$ and $S$ has a solution in $K$. As we said in the introduction, if $\mathfrak{X}$ is the class of all groups, then existentially closedness is the same as algebraically closedness.

The next lemma and theorem are proved in 5. for the class of all groups, but we give here the proofs again for the sake of completeness. Recall that a class of groups is called inductive, if it contains the union of any chain of its elements.

Lemma 1.1. Let $\mathfrak{X}$ be an inductive class of groups which is closed under the operation of taking subgroups. Let $G \in \mathfrak{X}$. Then there is a group $H \in \mathfrak{X}$ which contains $G$ and its order is at most $\max \left\{\aleph_{0},|G|\right\}$. Further, for any finite system $S$ of equations and in-equations over $G$, we have either of the following assertions:

1- $S$ has a solution in $H$.

2- For any extension $H \subseteq E \in \mathfrak{X}$, the system $S$ has no solution in $E$.

Proof. Suppose $\kappa=\max \left\{\aleph_{0},|G|\right\}$. Clearly the cardinality of the set of all finite systems of equations and inequations over $G$, is also at most $\kappa$. We suppose that this set is well-ordered as $\left\{S_{\alpha}\right\}_{\alpha}$, by the ordinals $0 \leq \alpha \leq \kappa$. Let $G_{0}=G$ and suppose that $G_{\gamma} \in \mathfrak{X}$ is already defined in such a way that $\left|G_{\gamma}\right| \leq \kappa$ and $\beta<\gamma$ implies $G_{\beta} \subseteq G_{\gamma}$. Let

$$
K_{\alpha}=\bigcup_{\gamma<\alpha} G_{\gamma} .
$$

Clearly, $K_{\alpha} \in \mathfrak{X}$ and $\left|K_{\alpha}\right| \leq \kappa^{2}=\kappa$. If $S_{\alpha}$ has no solution in any extension of $K_{\alpha}$, then we set $G_{\alpha}=K_{\alpha}$, otherwise there is a $E \in \mathfrak{X}$ which contains $K_{\alpha}$ and $S_{\alpha}$ has a solution, say $\bar{u}=\left(u_{1}, \ldots, u_{n}\right)$ in $E$ ( $n$ is the number of indeterminate in $S_{\alpha}$ ). Let

$$
G_{\alpha}=\left\langle K_{\alpha}, u_{1}, \ldots, u_{n}\right\rangle \leq E .
$$

Then $G_{\alpha} \in \mathfrak{X}$ and $\left|G_{\alpha}\right| \leq \kappa$. So, for any $\alpha<\kappa$, we have defined a $G_{\alpha}$. Note that, we have also

$$
\beta<\alpha \Rightarrow G_{\beta} \subseteq G_{\alpha} .
$$

Now, the group $H=\cup G_{\alpha} \in \mathfrak{X}$ has the required properties. This is because, any system $S=S_{\alpha}$ which has no solution in $H$, has no solution in $K_{\alpha}$ as well. So it has no solution in any extension of $H$.

Theorem 1.2. Let $\mathfrak{X}$ be an inductive class of groups which is closed under the operation of taking subgroups. Let $G \in \mathfrak{X}$. Then, there exists a group $G^{*} \in \mathfrak{X}$, with the following properties,

1 - $G$ is a subgroup of $G^{*}$.

2- $G^{*}$ is existentially closed in the class $\mathfrak{X}$.

3- $\left|G^{*}\right| \leq \max \left\{\aleph_{0},|G|\right\}$. 
Proof. Let $G^{0}=G$ and $G^{1}=H$, where $H$ is the group constructed in the lemma. Suppose $G^{m}$ is already defined and $G^{m+1}$ is the group which is proved to does exist for $G^{m}$ in the lemma. Let $G^{*}=\cup G^{m}$. Therefore, $G^{*} \in \mathfrak{X}$, satisfies conditions 1 and 3 . To prove 2 , suppose $S$ is a consistent system, with coefficients from $G^{*}$. Since $S$ is finite, so there is an $m$ such that all of the coefficients of $S$ belong to $G^{m}$. So, $S$ has a solution in $G^{m+1} \subseteq G^{*}$.

Note that in the number 3 of the above theorem, we may have $\left|G^{*}\right|<$ $\max \left\{\aleph_{0},|G|\right\}$. For example, suppose $G$ is an arbitrary group and $\mathfrak{X}$ is the set of all subgroups of $G$. Then trivially, $G^{*}=G$ and so, if $G$ is finite, then the inequality 3 is strict. Note that also, there are many inductive classes of groups, which are closed under subgroup: any variety, quasi-variety and in general, any universal class of groups has this property. As the first application of the above theorem, we show that every torsion free group can be embedded in a torsion free group with exactly two conjugacy classes. Note that we can use a similar arguments to prove the existence of torsion free groups of any infinite cardinality with just two conjugacy classes. Also note that the type of the group we are giving here, is not new, it is known from works of Higman, Neumann and Neumann [3].

Corollary 1.3. Every torsion free group $G$ can be embedded in a torsion free group $G^{*}$ which has just two conjugacy classes and its cardinality is the same as the cardinality of $G$.

Proof. Suppose $\mathfrak{X}$ is the class of all torsion free groups. Hence $\mathfrak{X}$ is inductive and closed under the operation of taking subgroups. We, begin with the group $G \in \mathfrak{X}$. Suppose $G^{*} \in \mathfrak{X}$ is the existentially closed group relative to $\mathfrak{X}$, which is constructed for $G$ in the theorem. We show that $G^{*}$ is the required group. Let $a, b \in G^{*}$ be two non-identity elements. Consider the equation $x a x^{-1}=b$. Let

$$
G_{a, b}^{*}=\left\langle G^{*}, t: t a t^{-1}=b\right\rangle
$$

be an HNN-extension of $G^{*}$. We know that every torsion element of this HNN-extension is conjugate to a torsion element of $G^{*}$, so $G_{a, b}^{*}$ is torsion free. It also contains $G^{*}$ as a subgroup and clearly $t$ is a solution for $x a x^{-1}=b$ in $G_{a, b}^{*}$. Therefor, there is already a solution in $G^{*}$. Hence $G^{*}$ is a torsion free group, containing $G$, with just two conjugacy classes. Further $\left|G^{*}\right|=$ $|G|$.

\section{An EMBEDDING THEOREM FOR $\mathrm{T}_{\pi}$-GROUPS}

It is easy to see that the simple group $G^{*}$ obtained in the corollary 1.3 is divisible. In this section, we prove a similar result for $\mathrm{T}_{\pi}$-groups. Let $\pi$ be a set of primes and $G$ be a group, such that its torsion elements have $\pi$-orders, i.e. the equality $x^{m}=1$ implies that $x=1$ or $m$ is a $\pi$-number. 
Then we say that $G$ is a $\mathrm{T}_{\pi}$-group. If $\pi=\emptyset$, then $\mathrm{T}_{\pi}$-groups are exactly torsion free groups. Note that a finite $\mathrm{T}_{\pi}$-group is just a finite $\pi$-group. A group $G$ is also said to be a $\pi^{\prime}$-group, if for any $\pi^{\prime}$-number $m$, every element of $G$ has an $m$-th root.

Theorem 2.1. Let $G$ be a $T_{\pi}$-group. Then there exists a $\pi^{\prime}$-divisible simple $T_{\pi}$-group $G^{*}$ containing $G$ and with the cardinality $\max \left\{\aleph_{0},|G|\right\}$, such that

1- element of the same order in $G^{*}$ are conjugate,

2- $G^{*}$ is not finitely generated,

3- every finite $\pi$-group embeds in $G^{*}$.

4- every finitely presented $\pi$-group can be residually embedded in $G^{*}$,

5- for any finite $\pi$-group $A$, we have $\operatorname{Aut}(A) \cong \frac{N_{G^{*}}(A)}{C_{G^{*}}(A)}$.

Proof. Let $\mathfrak{X}_{\pi}$ be the class of all $\mathrm{T}_{\pi}$-groups. This class is inductive and closed under subgroup. Since $G \in \mathfrak{X}_{\pi}$, so there exists a group $G^{*} \in \mathfrak{X}_{\pi}$ containing $G$, which is existentially closed in the class $\mathfrak{X}_{\pi}$. Suppose $1 \neq a, b \in G^{*}$ have the same orders. We show that $a$ and $b$ are conjugate. Suppose the HNN-extension

$$
G_{a, b}^{*}=\left\langle G^{*}, t: t a t^{-1}=b\right\rangle .
$$

It is enough to show that $G_{a, b}^{*}$ belongs to $\mathfrak{X}_{\pi}$. Let $x$ be an element of finite order in $G_{a, b}^{*}$. We know that $x$ is conjugate to an element of $G^{*}$, see [3]. So the order of $x$ is a $\pi$-number, showing that $G_{a, b}^{*} \in \mathfrak{X}_{\pi}$. This shows that elements of the same order in $G^{*}$ are conjugate. To show that $G^{*}$ is $\pi^{\prime}$ divisible, let $x \in G^{*}$ and $m$ be a $\pi^{\prime}$-number. We know that the orders of $x$ and $x^{m}$ are equal. Hence there is a $z \in G^{*}$ such that $x=z x^{m} z^{-1}$. Suppose $u=z x z^{-1}$. Then $u^{m}=x$ and hence $G^{*}$ is $\pi^{\prime}$-divisible.

We show that $G^{*}$ is simple. Note that $G^{*} *\langle x\rangle$ is a $\mathrm{T}_{\pi^{-} \text {-group. This follows }}$ from the fact that $G^{*}$ is an $\mathrm{T}_{\pi}$-group and the the reduced form of elements in free products is unique. Let $1 \neq a, w \in G^{*}$ be arbitrary elements and suppose $u=w x w^{-1} x^{-1}$ and $v=a x w^{-1} x^{-1}$. Then $u$ and $v$ are reduced in the free product and so they have infinite orders. Hence we can consider the HNN-extension

$$
M=\left\langle G^{*} *\langle x\rangle, t: t u t^{-1}=v\right\rangle .
$$

With the same argument (as for $G_{a, b}^{*}$ ), we see that $M$ is also an $\mathrm{T}_{\pi}$-group. The equation

$$
t w x w^{-1} x^{-1} t^{-1}=a x w^{-1} x^{-1}
$$

has a solution for $t$ and $x$ in $M$, therefore it has already a solution in $G^{*}$. We have

$$
a=\left(t w t^{-1}\right)\left(t x w^{-1} x^{-1} t^{-1}\right)\left(x w^{-1} x^{-1}\right),
$$

so $a \in\left\langle w^{G^{*}}\right\rangle$. Hence, for all $1 \neq w \in G^{*}$, we have $G^{*}=\left\langle w^{G^{*}}\right\rangle$. This proves that $G^{*}$ is simple.

Now, since $G^{*}$ is non-abelian simple group, we have $Z\left(G^{*}\right)=1$. On the other hand, for any finite subset $a_{1}, \ldots, a_{m} \in G^{*}$, the system

$$
a_{1} x=x a_{1}, \ldots, a_{m} x=x a_{m}, x \neq 1,
$$


has a solution in the $\mathrm{T}_{\pi^{-} \text {-group }} G^{*} \times\langle x\rangle$, so it has a solution in $G^{*}$. Therefore

$$
C_{G^{*}}\left(\left\langle a_{1}, \ldots, a_{m}\right\rangle\right) \neq 1
$$

This proves that $G^{*}$ is not finitely generated.

Now, suppose that $A$ is a finite $\pi$-group and

$$
A=\left\{1=g_{0}, g_{1}, \ldots, g_{m}\right\}
$$

For any $1 \leq i, j \leq m$ there exists a unique $0 \leq k(i, j) \leq m$, such that $g_{i} g_{j}=g_{k(i, j)}$. The group $G^{*} \times A$ is clearly an $\mathrm{T}_{\pi^{-} \text {-group and the system }}$

$$
x_{i} x_{j}=x_{k(i . j)} ;(0 \leq i, j \leq m) ; x_{i} \neq x_{j} ;(0 \leq i<j \leq m)
$$

has a solution in $G^{*} \times A$ and hence it has a solution in $G^{*}$. This shows that $A$ is embedded in $G^{*}$. To prove 4 , let $A$ be a finitely presented $\pi$-group, with a finite presentation

$$
A=\left\langle x_{1}, \ldots, x_{n}: r_{1}, \ldots, r_{m}\right\rangle .
$$

Suppose $1 \neq w \in A$ and consider the system

$$
r_{i}\left(x_{1}, \ldots, x_{n}\right)=1 ;(1 \leq i \leq m), w\left(x_{1}, \ldots, x_{n}\right) \neq 1 .
$$

Clearly this equation has a solution in $G^{*} \times A \in \mathfrak{X}_{\pi}$, and hence it has a solution in $G^{*}$. This proves that there is a homomorphism $\varphi: A \rightarrow G^{*}$ with $\varphi(w) \neq 1$. Hence $A$ embeds in $G^{*}$ residually. We now prove 5 . Suppose again that $A$ is a finite $\pi$-group and $\alpha \in \operatorname{Aut}(A)$. This time, we consider the system

$$
x a x^{-1}=\alpha(a) ;(a \in A) .
$$

This system has a solution in the HNN-extension

$$
G_{\alpha}^{*}=\left\langle G^{*}, t: t a t^{-1}=\alpha(a) ;(a \in A)\right\rangle .
$$

Therefore, there exists an element $x \in G^{*}$ such that $\alpha$ is equal to the restriction of the inner automorphism $T_{x}$ to $A$. This also shows that $x \in N_{G^{*}}(A)$ and so the map which sends $x$ to the restriction of $T_{x}$ on $A$ is an epimorphism from $N_{G^{*}}(A)$ to $A u t(A)$ with the kernel $C_{G^{*}}(A)$. This completes the proof of 5 .

We can generalize the above theorem for HNN-classes of groups. A class $\mathfrak{X}$ of groups is called an HNN-class if it is inductive, closed under subgroup, and HNN-extensions of the elements of $\mathfrak{X}$ belong to $\mathfrak{X}$. The class of all $\mathrm{T}_{\pi}$-groups is an example of HNN-classes. By a similar argument as in the above theorem, we can prove the next result.

Theorem 2.2. Let $\mathfrak{X}$ be an $H N N$-class of groups. Then every element $G$ of $\mathfrak{X}$ embeds in a simple $G^{*} \in \mathfrak{X}$, with the cardinality at most $\max \left\{\aleph_{0},|G|\right\}$. Further elements of the same orders in $G^{*}$ are conjugates. 


\section{Some OlshanskiI Like GROUPS}

In mid twenties, Alfred Tarski asked about the existence of infinite groups all proper non-trivial subgroups of which are of fixed prime order $p$. In 1982, A. Yu. Olshanskii [4, constructed an uncountable family of such groups using his geometric method of graded diagrams over groups, for all primes $p>10^{75}$. The groups constructed are called Tarskii monsters since then. These groups are two-generator simple groups and hence are countable. In this section, for any fixed prime $p$, we give a quite elementary proof for existence of countable non-abelian simple groups with the property that their all non-trivial finite subgroups are cyclic of order $p$.

We will consider two special classes of groups in this section. The first one consists of groups all finite subgroups in which are cyclic. We will denote this class by $\mathfrak{X}_{f c}$. The second class which will be denoted by $\mathfrak{X}_{p}$, is the class of all groups in which their non-trivial finite subgroups are of order $p$, for a fixed prime $p$. Note that both classes are HNN-classes. Clearly the Monsters constructed by Olshanskii satisfy the requirements of the next theorem, but we don't use that monsters, since we have a very elementary proof for our claims. What we need is the theorem 1.2 and some facts about finite subgroups of HNN-extensions (and also those of free products). It is known that (see [3], page 212) every finite subgroup of any HNN-extension

$$
G=\left\langle A, t: t F t^{-1}=\phi(F)\right\rangle
$$

is contained in some conjugate of $A$. Also, every finite subgroup of any free product $A * B$ is contained in some conjugate of $A$ or some conjugate of $B$.

Theorem 3.1. There exists a countable non-abelian simple group $M$ such that all finite subgroups of $M$ are cyclic and for any prime $p$, the group $M$ has an element of order $p$.

Proof. We know that the class $\mathfrak{X}_{f c}$ is inductive and closed under subgroup, so we can apply 1.2. Note that, we have many groups which belong to $\mathfrak{X}_{f c}$, for example any finite cyclic group, $\mathbb{Z}_{p^{\infty}}$, and any torsion free group. So, let $G \in \mathfrak{X}_{f c}$ be a countable arbitrary element. Therefore, there exists a group $G^{*}$ satisfying requirements of 1.2 . Let $M=G^{*}$ and $p$ be a prime. We show that $M$ has an element of order $p$. Suppose this is not true. Hence the system

$$
x^{p}=1, x \neq 1
$$

has no solutions in $M$. Let $H \leq M \times \mathbb{Z}_{p}$ be a finite subgroup. We have $H \subseteq \pi_{1}(H) \times \pi_{2}(H)$, where $\pi_{1}$ and $\pi_{2}$ are projections. Since $M \in \mathfrak{X}_{f c}$, so $\pi_{1}(H)$ is cyclic and by our assumption $p$ is co-prime to the order of $\pi_{1}(H)$. Therefore $\pi_{1}(H) \times \pi_{2}(H)$ is cyclic and so is $H$. Hence $M \times \mathbb{Z}_{p}$ belongs to $\mathfrak{X}_{f c}$. But the above system has a solution in $M \times \mathbb{Z}_{p}$, a contradiction.

Note that, for any cyclic group $\langle x\rangle$ the group $M *\langle x\rangle$ belongs to $\mathfrak{X}_{f c}$. To see this, let $H$ be a finite subgroup of this free product. Then $H$ must be contained in a conjugate of $M$ and hence $H$ is cyclic. Now, suppose 
$1 \neq a, b \in M$ and consider two elements

$$
u=a x a^{-1} x^{-1}, v=b x a^{-1} x^{-1}
$$

in $M *\langle x\rangle$. Clearly $u$ and $v$ have infinite orders and so we can consider the HNN-extension

$$
M^{*}=\left\langle M *\langle x\rangle, t: t u t^{-1}=v\right\rangle .
$$

Let $H$ be a finite subgroup of this HNN-extension. Then $H$ is contained in some conjugate of $M$ and therefore it is cyclic. Hence $M^{*}$ is also an element of $\mathfrak{X}_{f c}$. So, the equation

$$
\operatorname{taxa}^{-1} x^{-1} t^{-1}=b x a^{-1} x^{-1}
$$

has a solution for $t$ and $x$ in $M^{*}$, therefore it has already a solution in $M$. We have

$$
b=\left(t a t^{-1}\right)\left(t x a^{-1} x^{-1} t^{-1}\right)\left(x a^{-1} x^{-1}\right),
$$

so $b \in\left\langle a^{M}\right\rangle$. Hence, for all $1 \neq a \in M$, we have $M=\left\langle a^{M}\right\rangle$. This proves that $M$ is simple.

Finally note that since for any $a \in M$, the in-equation $a x \neq x a$ has a solution in $M *\langle x\rangle$, so the center of $M$ is trivial and hence $M$ is nonabelian.

Now, a similar argument on the class $\mathfrak{X}_{p}$ proves the next theorem.

Theorem 3.2. Let $p$ be a fixed prime. Then there exists a countable nonabelian simple group $M$ (which is not torsion free) such that any finite nontrivial subgroup of $M$ is cyclic of order $p$.

The following two corollaries can be deduced instantly.

Corollary 3.3. There exists a non-abelian two generator group such that the orders of the generators are distinct primes and its every finite subgroup is cyclic.

Corollary 3.4. There exists a non-abelian p-group with two generators such that its every finite subgroup has order $p$.

\section{Existentially Closed algebras}

We are going now to find similar embedding theorems for Lie algebras. But there are two major differences between groups and Lie algebras. First, we don't have any suitable definition of torsion in the case of Lie algebras so, in advance, we don't have a parallel concept of $\mathrm{T}_{\pi}$-Lie algebra and so on. Instead, we can express our theorems in terms of arbitrary Lie algebras. The second main difference is related to HNN-extensions of Lie algebras. Here, an HNN-extension comes from a Lie algebra and a derivation of some subalgebra, despite groups where HNN-extensions are always defined by groups and isomorphisms between subgroups. We will give a brief summary of HNN-extensions of Lie algebras in the next section. In this section, we give the analogue of Theorem 1.2 for Lie algebras, in fact since it can be 
formulated for arbitrary non-associative algebras, we prove it in the most general form.

Lemma 4.1. Let $\mathfrak{X}$ be an inductive class of (not necessarily associative) algebras over a field $K$. Suppose $\mathfrak{X}$ is closed under subalgebra and $L \in \mathfrak{X}$. Then there exists an algebra $H \in \mathfrak{X}$ containing $L$ such that its dimension is at most

$$
\max \left\{\aleph_{0}, \operatorname{dim} L,|K|\right\} .
$$

Further, for any system $S$ of equations and in-equations over $L$, we have either of the following assertions:

1- $S$ has a solution in $H$.

2- For any extension $H \subseteq E \in \mathfrak{X}$, the system $S$ has no solution in $E$.

Proof. We assume that $X$ is a countable set of variables and

$$
\eta=\max \left\{\aleph_{0}, \operatorname{dim} L,|K|\right\} .
$$

Any equation over $L$ consists of finitely many elements of $L$ and $X$, so $\kappa$, the number of systems of equations and in-equations over $L$, is $|L \cup X|$. Note that $|L|=\max \{\operatorname{dim} L,|K|\}$, hence $\kappa=|L|+\aleph_{0}=\eta$.

We well-order the set of all systems as $\left\{S_{\alpha}\right\}_{\alpha}$, using ordinals $0 \leq \alpha \leq \kappa$. Suppose $L_{0}=L$. Let for any $0 \leq \gamma \leq \alpha$, the algebra $L_{\gamma} \in \mathfrak{X}$ is defined in such a way that $\left|L_{\gamma}\right| \leq \kappa$ and

$$
\beta \leq \gamma \Rightarrow L_{\beta} \subseteq L_{\gamma}
$$

We put

$$
E_{\alpha}=\bigcup_{\gamma \leq \alpha} L_{\gamma}
$$

so $E_{\alpha} \in \mathfrak{X}$ and further

$$
\left|E_{\alpha}\right| \leq \alpha\left|L_{\gamma}\right| \leq \kappa^{2}=\kappa
$$

Suppose $S_{\alpha}$ has not solution in any extension of $E_{\alpha}$. Then we set $L_{\alpha}=E_{\alpha}$. If there is an extension $E_{\alpha} \subseteq E \in \mathfrak{X}$ such that $S_{\alpha}$ has a solution $\left(u_{1}, \ldots, u_{n}\right)$ in $E$ ( $n$ is the number of indeterminate in $S_{\alpha}$ ), then we set

$$
L_{\alpha}=\left\langle E_{\alpha}, u_{1}, \ldots, u_{n}\right\rangle \subseteq E .
$$

Since $\mathfrak{X}$ is closed under subalgebra, so $L_{\alpha} \in \mathfrak{X}$ and also

$$
\left|L_{\alpha}\right|=\left|E_{\alpha}\right| \leq \kappa \text {. }
$$

Now, we define

$$
H=\bigcup_{0 \leq \alpha \leq \kappa} L_{\alpha}
$$

which is an element of $\mathfrak{X}$. We have $|H| \leq \kappa^{2}=\kappa$ and hence

$$
\max \{\operatorname{dim} H,|K|\} \leq \max \left\{\aleph_{0}, \operatorname{dim} L,|K|\right\},
$$

therefore we have

$$
\operatorname{dim} H \leq \max \left\{\aleph_{0}, \operatorname{dim} L,|K|\right\}
$$


Theorem 4.2. Let $\mathfrak{X}$ be an inductive class of (not necessarily associative) algebras over a field $K$. Suppose $\mathfrak{X}$ is closed under subalgebra and $L \in \mathfrak{X}$. Then there exists an algebra $L^{*} \in \mathfrak{X}$ with the following properties,

1 - $L$ is a subalgebra of $L^{*}$.

2- $L^{*}$ is existentially closed in the class $\mathfrak{X}$.

3- $\operatorname{dim} L^{*} \leq \max \left\{\aleph_{0}, \operatorname{dim} L,|K|\right\}$.

Proof. Let $H^{0}=L$ and $H^{1}=H$ be an algebra satisfying requirements of the previous lemma. Suppose $H^{m}$ is defined and let $H^{m+1}$ be an algebra obtained by the lemma from $H^{m}$. We have

$$
\begin{aligned}
\operatorname{dim} H^{m+1} & \leq \max \left\{\aleph_{0}, \operatorname{dim} H^{m},|K|\right\} \\
& =\max \left\{\aleph_{0}, \operatorname{dim} L,|K|\right\} .
\end{aligned}
$$

Now, suppose

$$
L^{*}=\bigcup_{m} H^{m} .
$$

This is an algebra having the properties 1-2-3.

\section{EmbedDing of Lie AlgEBRAS}

In 2 and [7, the concept of the HNN-extension is defined for Lie algebras. Suppose $L$ is a Lie algebra over a filed $K$ and $A$ is a subalgebra. Let $\delta: A \rightarrow L$ be a derivation. Define a Lie algebra $L_{\delta}$ with the presentation

$$
L_{\delta}=\langle L, t:[t, a]=\delta(a) ;(a \in A)\rangle .
$$

The properties of this HNN-extension is studied in [2] and [7]. It is proved that $L$ is a subalgebra of $L_{\delta}$. Similar constructions are also introduced for Lie $p$-algebras and rings in [2. In this section, using this HNN-extension and the notion of existentially closed Lie algebras, we obtain a new embedding theorem.

Theorem 5.1. Let $L$ be a Lie algebra over a field $K$. Then there exists a Lie algebra $L^{*}$ having the following properties,

1 - $L$ is a subalgebra of $L^{*}$,

2- for any non-zero $a, b \in L^{*}$, there exists $x \in L^{*}$ such that $[x, a]=b$, and so $L^{*}$ is simple.

3- $\operatorname{dim} L^{*} \leq \max \left\{\aleph_{0}, \operatorname{dim} L,|K|\right\}$,

4- $L^{*}$ is not finitely generated,

5- every finite dimensional simple Lie algebra over $K$ embeds in $L^{*}$,

6 - every finitely presented Lie algebra over $K$ embeds residually in $L^{*}$,

7 - if $K$ is finite, then every finite dimensional Lie algebra over $K$ embeds in $L^{*}$,

8- if $K$ is finite and $A$ is finite dimensional Lie algebra over $K$, then we have

$$
\operatorname{Der}(A) \cong \frac{N_{L^{*}}(A)}{C_{L^{*}}(A)}
$$


Proof. We suppose that $\mathfrak{X}$ is the class of all Lie algebras and then we apply the theorem 3.2. Hence, there exists an existentially closed Lie algebra $L^{*}$ containing $L$ such that

$$
\operatorname{dim} L^{*} \leq \max \left\{\aleph_{0}, \operatorname{dim} L,|K|\right\} .
$$

Let $0 \neq a, b \in L^{*}$ and $\delta:\langle a\rangle \rightarrow L$ be the derivation $\delta(a)=b$. Consider the HNN-extension

$$
L_{\delta}^{*}=\left\langle L^{*}, t:[t, a]=b\right\rangle .
$$

We know that $L^{*} \leq L_{\delta}^{*}$ and the equation $[x, a]=b$ has a solution in $L_{\delta}^{*}$, so 2 is proved. This implies also that $L^{*}$ is simple. To prove 4 , suppose $x_{1}, \ldots, x_{n}$ is a finite set of elements of $L^{*}$. Consider the system

$$
\left[x, x_{i}\right]=0 ;(1 \leq i \leq n), x \neq 0 .
$$

This system has a solution in the Lie algebra $L^{*} \times\langle x\rangle$, and so we have $C_{L^{*}}\left(\left\langle x_{1}, \ldots, x_{n}\right\rangle\right) \neq 0$, while $Z\left(L^{*}\right)=0$. Therefore $L^{*}$ is not finitely generated.

Suppose $E$ is a finite dimensional simple Lie algebra with a basis $u_{1}, \ldots, u_{n}$ and suppose $\left[u_{i}, u_{j}\right]=\sum_{r} \lambda_{i j}^{r} u_{r}$. Consider the system

$$
\left[x_{i}, x_{j}\right]=\sum_{r} \lambda_{i j}^{r} x_{r} ;(1 \leq i, j \leq n), x_{i} \neq 0 ;(1 \leq i \leq n) .
$$

This system has a solution in $L^{*} \times E$ and so there is a non-zero homomorphism $E \rightarrow L^{*}$. Therefore $E$ embeds in $L^{*}$. The proof of 6 is similar, so we prove 7 . Suppose $K$ is finite and let $E$ be a finite dimensional Lie algebra with a basis $u_{1}, \ldots, u_{n}$ and structural constants $\lambda_{i j}^{r}$, i.e. $\left[u_{i}, u_{j}\right]=\sum_{r} \lambda_{i j}^{r} u_{r}$. Let $T$ be the set of all $n$-tuples $\left(a_{1}, \ldots, a_{n}\right) \in K^{n}$ with $a_{i} \neq 0$ for some $i$. Consider the system

$$
\begin{gathered}
{\left[x_{i}, x_{j}\right]=\sum_{r} \lambda_{i j}^{r} x_{r} ;(1 \leq i, j \leq n)} \\
\sum_{r} a_{r} x_{r} \neq 0 ;\left(\left(a_{1}, \ldots, a_{n}\right) \in T\right) .
\end{gathered}
$$

This system has a solution in $L^{*} \times E$, and so $E$ embeds in $L^{*}$.

Finally, to prove 8, let $K$ be finite and $A$ be finite dimensional (subalgebra of $\left.L^{*}\right)$. Let $\delta \in \operatorname{Der}(A)$ and consider the HNN-extension

$$
L_{\delta}^{*}=\left\langle L^{*}, t:[t, a]=\delta(a) ;(a \in A)\right\rangle
$$

in which the system

$$
[x, a]=\delta(a) ;(a \in A)
$$

has a solution. So, there is an $x \in L^{*}$ such that $\delta(a)=[x, a]$ for all $a \in A$. Clearly $x \in N_{L^{*}}(A)$. So there is an epimorphism $N_{L^{*}}(A) \rightarrow \operatorname{Der}(A)$ with the kernel $C_{L^{*}}(A)$. So we have

$$
\operatorname{Der}(A) \cong \frac{N_{L^{*}}(A)}{C_{L^{*}}(A)}
$$


As an interpretation of the above theorem, define a Lie algebra to be division Lie algebra, if for all $a$ and $b$ with $a \neq 0$, there exists an $x$ such that $[a, x]=b$. Then the above theorem shows that every Lie algebra can be embed in a simple division Lie algebra. However, that division Lie algebra is not finitely generated. It can be asked that if there exists a finitely generated division Lie algebra.

In 6], we proved that if a Lie algebra $L$ over a filed of characteristics zero has a finite dimensional ideal $U$ such that $U^{n} \subseteq[A, U]$ for some abelian subalgebra $A$ and $n \geq 2$, then $U$ is solvable. Here $[A, U]$ is the linear span of the set $\{[a, x]: a \in \bar{A}, x \in U\}$. The Lie algebra $L^{*}$ which we obtained in 5.1, shows that the assumption on the dimension of $U$ is essential, for example of we let $U=L^{*}$ and if we assume that $A$ is any 1-dimensional subalgebra, then always $U^{n} \subseteq[A, U]$, but clearly $U$ is not solvable ( $L^{*}$ is simple).

\section{Existentially COMPLETE GROUPS}

In this section, we define the notion of existentially complete groups. It can be define the general frame of the universal algebra. Existentially complete groups are special kind of existentially closed groups, where in their definition, we use some special sentences of the second order language of groups.

Let $G$ be a group and $A \leq G$ be a finitely generated subgroup. Suppose $w\left(x_{1}, \ldots, x_{n}\right) \in G * F\left(x_{1}, \ldots, x_{n}\right)$. The expression

$$
w\left(x_{1}, \ldots, x_{n}\right) \in A
$$

is called a membership. The negation of a membership is a non-membership. We say that a finite system of memberships and non-memberships (briefly, a membership system), is consistent, if a there is a group $K$ containing $G$, such that the system has a solution in $K$. A group $G$ with the property that every consistent membership system has a solution in $G$, will be called existentially complete. Clearly, such a group is in also existentially closed. We can also define the notion of existentially complete group in a given class. In some classes, the two notions are equivalent, for example, in the class of locally finite groups, there is no difference between two notions. We can define similarly, existentially complete Lie algebras and also it is easy to see that over finite fields, two properties of existentially closedness and existentially completeness are equivalent.

A completely similar argument as in the section 1 , the reader can prove the next theorem. As a result, it shows that there exists existentially complete groups.

Theorem 6.1. Let $\mathfrak{X}$ be an inductive class of groups, closed under subgroup, such that $G \in \mathfrak{X}$. Then there exists an existentially complete group $G^{*}$, containing $G$, such that

$$
\left|G^{*}\right| \leq \max \left\{\aleph_{0},|G|\right\}
$$


The following embedding theorem, gives an interesting application of the notion of existentially complete groups.

Theorem 6.2. Let $M$ be an existentially complete group and $A$ be a finitely generated subgroup. Then, every extension of $A$ by any finite group embeds in $M$.

Proof. Let $A=\left\langle a_{1}, \ldots, a_{m}\right\rangle$ and $G$ be a group, having a normal subgroup $B$, isomorphic to $A$. Let $\phi$ is the isomorphism from $A$ to $B$ and $b_{i}=\phi\left(a_{i}\right)$. Suppose also that

Let

$$
\frac{G}{B}=\left\{g_{1} B, \ldots, g_{n} B\right\}
$$

$$
\left(g_{i} B\right)\left(g_{j} B\right)=g_{r(i, j)} B
$$

where $r(i, j)$ is a function of $i$ and $j$. So, for any $i$ and $j$, there exists an element $q_{i j} \in B$ such that

$$
g_{r(i, j)}^{-1} g_{i} g_{j}=q_{i j}
$$

We also have $g_{i} b_{j} g_{i}^{-1} \in B$, so $g_{i} b_{j} g_{i}^{-1}=p_{i j}$, for some $p_{i j} \in B$. Now, consider the next membership system

$$
\begin{aligned}
x_{r(i, j)}^{-1} x_{i} x_{j} & =\phi^{-1}\left(q_{i j}\right), & & (1 \leq i, j \leq n) \\
x_{i}^{-1} x_{j} & \notin A, & & (1 \leq i<j \leq n) \\
x_{i} a_{j} x_{i}^{-1} & =\phi^{-1}\left(p_{i j}\right), & & (1 \leq i \leq n, 1 \leq j \leq m)
\end{aligned}
$$

Let $M_{1}=\langle M * G: A=B, \phi\rangle$ be the amalgamated free product of $M$ and $G$. We can verify easily that $\left(g_{1}, \ldots, g_{n}\right)$ is a solution of the above system in $M_{1}$. Hence, the system has a solution $\left(u_{1}, \ldots, u_{n}\right)$ in $M$. Let

$$
K=\left\langle a_{1}, \ldots, a_{m}, u_{1}, \ldots, u_{n}\right\rangle,
$$

which is a subgroup of $M$, containing $A$ as a normal subgroup, by (3). This shows that every element of $K$ has the form $w\left(u_{1}, \ldots, u_{n}\right) a$, such that $a \in A$ and $w$ is a group word in $u_{1}, \ldots, u_{n}$. Now, by (1), we can replace every $u_{i} u_{j}$ by $u_{r(i, j)} \phi^{-1}\left(q_{i j}\right)$, and collecting all factors by this method, we finally see that any element of $K$ has the unique form $u_{i} a$ for some $1 \leq i \leq n$ and $a \in A$. Note that the same observation is also true for the elements of $G$. Now, we prove that $K \cong G$. Let $\psi: K \rightarrow G$ be define as $\psi\left(u_{i} a\right)=g_{i} \phi(a)$. Clearly, $\psi$ is a bijection, so we show that it is a homomorphism.

Let $k, k^{\prime} \in K$. We have $k=u_{i} a$ and $k^{\prime}=u_{j} a^{\prime}$, for some $1 \leq i, j \leq n$ and $a, a^{\prime} \in A$. We have

$$
\psi\left(k k^{\prime}\right)=\psi\left(u_{i} u_{j}\left(u_{j}^{-1} a u_{j}\right) a^{\prime}\right) .
$$

Note that by (3), we can write $u_{j}^{-1} a u_{j}$ as $\phi^{-1}(p)$, for some suitable $p \in B$. Also by (1), we have $u_{i} u_{j}=u_{r(i, j)} \phi^{-1}\left(q_{i j}\right)$, hence

$$
\begin{aligned}
\psi\left(k k^{\prime}\right) & =\psi\left(u_{r(i, j)} \phi^{-1}\left(q_{i j}\right) \phi^{-1}(p) a^{\prime}\right) \\
& =g_{r(i, j)} q_{i j} p \phi\left(a^{\prime}\right) .
\end{aligned}
$$


On the other hand, we have

$$
\begin{aligned}
\psi(k) \psi\left(k^{\prime}\right) & =g_{i} \phi(a) g_{j} \phi\left(a^{\prime}\right) \\
& =g_{i} g_{j}\left(g_{j}^{-1} \phi(a) g_{j}\right) \phi\left(a^{\prime}\right) \\
& =g_{i} g_{j} p \phi\left(a^{\prime}\right) \\
& =g_{r(i, j)} q_{i j} p \phi\left(a^{\prime}\right) .
\end{aligned}
$$

This completes the proof.

As a final note, we should say that the same result also can be proved for Lie algebras.

Acknowledgement The author would like to thank M. Kuzucuoglu for comments and suggestions.

\section{REFERENCES}

[1] Higman, G., Scott, E. L. Existentially closed groups, Clarendon Press, 1988.

[2] Lichtman, A. I., Shirvani, M. HNN-extensions for Lie algebras, Proc. AMS, Vol. 125, No. 12, pp. 3501-3508, 1997.

[3] Lyndon, R. C., Schupp, P. E. Combinatorial group theory, Springer-Verlag, 2001.

[4] Olshanskii, A. Y. Geometry of defining relations in groups, Kluwer Academic Publishers, 1991.

[5] Scott, W. R. Algebraically closed groups, Proc. of AMS, No. 2, pp. 118-121, 1951.

[6] Shahryari, M. A note on derivations of Lie algebras, Bull. Aust. Math. Soc. Vol. 84, pp. 444-446, 2011.

[7] Wasserman, A. A derivation HNN construction for Lie algebras, Israil J. Math. Vol. 106, pp. 76-92, 1998.

Department of Pure Mathematics, Faculty of Mathematical Sciences, UniVERSITY OF TABRIZ, TABRIZ, IRAN

E-mail address: mshahryari@tabrizu.ac.ir 